\title{
Relationship between life of mate butterflies and leading selection characteristics
}

\author{
$U$. Khudayberdieva $^{1}, S$. Navruzov $^{1}, N$. Rajabov $^{1, *}, O$. Karimov $^{1}$, and $K H$. Fozilova $^{1}$ \\ ${ }^{1}$ Tashkent State Agrarian University, University str., 2, Tashkent province, Uzbekistan, 100140
}

\begin{abstract}
This article examines the history of silkworm breeding, the role of silkworm breeding in the national economy of the Republic of Uzbekistan and the role of silkworm breeding in the development of the industry. This is because the interrelationship of traits of economic value is of great importance in selection and breeding work. The experiments were conducted in 2015-2017 at the Silk Research Institute of Uzbekistan. The life expectancy of the experimental butterflies was 10.9-12.8 days and the variability was $33.9-56.6 \%$. The coefficient of variability indicates the degree of diversity in the population on this trait. Thus, it is clear from the results that the population of "Marvarid" and "Liniya 27 " has the ability to carry out selection work on the sign of life expectancy of female butterflies.
\end{abstract}

\section{Introduction}

Silkworm breeding has long been a thriving industry and is now one of the most important sectors of agriculture. Silk raw materials are widely used in textiles, medicine, aviation, aerospace, radio engineering, as well as products made of silk and silk products have a high export potential $[1,2]$.

The Republic of Uzbekistan is a world leader in the continuous improvement of silkworm care and cocoon production, the widespread introduction of effective methods of production and deep processing of cocoons, raw silk, silk yarn, the production of finished silk products, increasing the export potential of the industry $[3,7]$. To date, the volume of cocoon production in the country has reached almost 20,000 tons. The demand for silk products that meet the requirements of the world market is growing day by day. This, in turn, requires the cultivation of high-yielding, silky and technologically advanced breeds and hybrids of silkworms in the cultivation of high-quality and abundant cocoons. If the breeds and industrial hybrids suitable for each region are created and introduced, it will be possible to grow high-quality quality cocoons $[7,8]$.

The role of new scientifically based methods of selection and breeding work in the creation of new high-yielding and high-viability breeds is enormous $[4,5]$. It should be noted that the leading economic value traits of mulberry silkworm appear under the influence of polygenes. Therefore, at each stage of breeding, it is necessary to select the strongest genotypes for the offspring during the selection process and to increase the

\footnotetext{
* Corresponding author: rajabovn7784@gmail.com
} 
performance of the breeding material from generation to generation. Otherwise, there will be a decrease in productivity and vitality in the population of the breed [6].

In silkworm breeding and breeding, the viability of the offspring, cocoon productivity has always been in the focus of breeders, and in order to create any new breeding systems, it is necessary to first study the correlation, variability and hereditary characteristics of traits and characteristics and creates a selection plan based on these parameters $[9,10]$.

One of the main tasks of our research is to determine the degree of correlation between butterfly life expectancy and leading farm value traits. During the selection and breeding work, the correlation between the characters of economic value is of great importance. This is because the selection process performed on a particular character should not lead to a decrease in another secondary character. In addition, knowledge of the interrelationships of important traits is of great scientific and practical importance in the development of selection programs and the correct determination of selection methods $[10,11]$.

A number of scientific studies have been conducted by many researchers aimed at improving the technology of silkworm seed preparation. Among the methods used in the breeding and breeding of mulberry silkworms, the most effective method is selection. The result of individual or group selection of quantitative traits depends on the variability, heredity of traits in the population, and the degree of their correlation [10-12].

The main parameters of the variable include the arithmetic mean $(\mathrm{m})$, the error of the arithmetic mean $(\mathrm{S})$, the standard deviation $(\sigma)$, and the coefficients of variation $(\mathrm{Cv})$. In the scientific literature, one can find a lot of information about the fact that the availability of choice in terms of productivity and fertility of farm animals depends on their variability. In particular, the variability of productivity and technological characteristics of mulberry silkworm has been widely covered in research work on genetics and selection [13, 14].

It was determined the coefficients of variability of fertility traits of some breeds: 8 breeds of SANIISH egg weight $\mathrm{Cv}=5.0-31.5 \%$; SAN $=9-26 \mathrm{Cv}=1.5-26.5 \%$; $\mathrm{Cv}=2.0$ $52.0 \%$ in the EP breed. Based on these variability coefficients, the author assessed the degree of homogeneity of the egg laying of the strains [10].

The Azad and Gyanja breeds were found to be dependent on the intensity of selection differential selection based on the variability of the number and weight of eggs in the egg laying hen [11].

U. N. Nasirillaev [7, 8] for several years studied the variability of cocoon productivity traits in superelite and elite populations at the Fergana breeding station (Table 1).

Table 1. Weight and silk productivity (silkability) of silkworms

\begin{tabular}{|c|c|c|c|}
\hline Breeds & $\begin{array}{c}\text { Silkworm } \\
\text { weight } \\
\text { Cv, \% }\end{array}$ & $\begin{array}{c}\text { Silkworm } \\
\text { cocoon } \\
\text { weight Cv, } \\
\mathbf{\%}\end{array}$ & $\begin{array}{c}\text { Silkability Cv, } \\
\text { \% }\end{array}$ \\
\hline From 5 months & 11.9 & 14.6 & 10.2 \\
\hline SANIISH 17 & 12.5 & 12.6 & 12.2 \\
\hline SANIISH 21 & 12.9 & 12.3 & 9.2 \\
\hline SANIISH 30 & 12.5 & 14.0 & 11.5 \\
\hline
\end{tabular}

According to the results of the experiment, the coefficient of variability of Tashkent 1 breed is $3.3-3.4 \%$ in terms of viability, $4.2-7.3 \%$ in terms of cocoon weight, $2.2-10.0 \%$ in terms of cocoon shell [12]. However, the highest variability was detected in the sign of butterfly emergence from the cocoon $(41.6-52.3 \%)$. That is, the variability of this sign is wide and there is diversity in the population [14].

The effectiveness of selection in mulberry silkworm selection largely depends on the degree of interdependence of the characters. If the selection traits are positively correlated 
with each other, then the performance of the second trait may also increase as a result of selection. However, if the correlation is negative or in the opposite direction, then a decrease in the index of the second character is likely to be observed [10-12].

Certain results have been obtained on determining the correlation between mulberry silkworm traits and their use in the selection process. In particular, the interdependence of butterfly germination and viability traits is of great importance for the practice of selection and breeding work. B. Nasirillaev [7] calculated the correlation coefficients between the physiological defect in egg laying and the viability of worms $(r=0.316)$. In his next research [8], he proved that there is a close correlation between egg resuscitation and worm viability in high-temperature incubation. Based on the connection between these traits, $\mathrm{N}$. Navruzov [10] managed to create Turkmenistan 3 and Turkmenistan 4 breeds suitable for the hot climatic conditions of Turkmenistan.

S. Navruzov [12] found a high correlation between butterfly germination and worm viability. His work was devoted to determining the degree of interdependence of important productivity traits such as cocoon weight, cocoon shell weight, and silkiness. Timely experiments have shown that the degree of dependence of these traits can vary depending on the area of care of the worms and the amount of feed.

In the breeding and seed enterprises, the work on the separation of breeding cocoons into rocks remains a topical issue. Therefore, it is difficult to call industrial seeds $100 \%$ hybrid seeds. However, in the science of silkworm breeding, in particular, geneticists and breeders of the Republic have created sex-marked breeds during ovulation and larvae, which allow to produce $100 \%$ hybrids, while parthenogenetic clones have been obtained that their offspring will be $100 \%$ female and there will be no problem of sex.

U. Umarova [13] highlighted the results of effect on the performance of the next generation by repeated crossbreeding of thin silkworm male butterflies. In the obtained results, live male silkworm cocoon was bred and their technological parameters were analyzed. The results of the study proved that multiple crossbreeds of genotypes with high technological performance did not adversely affect cocoon weight. In the second year of the experiment, it was found that the silk of the cocoons increased by 1.3 as compared to the comparator.

\section{Materials and methods}

The experiments were carried out in 2015-2017 in special worms of the laboratory "Breeding" of the Silk Research Institute. The experimental worms were incubated at a temperature of $24-25^{\circ} \mathrm{C}$ and a relative humidity of $70-75 \%$. After 10 days of incubation, the worms were raised and kept at a temperature of $26-27^{\circ} \mathrm{C}$ in young and $24-25^{\circ} \mathrm{C}$ in adults. When feeding worms with mulberry leaves, they were cared for at the expense of $1,200 \mathrm{~kg}$ of leaves per 1 box. The experimental breeds were fed with mulberry leaves belonging to the Silk Research Institute of Uzbekistan [14].

For the experiments, a large cocoon round cocoon-shaped "Marvarid" breed of mulberry silkworm and a medium-cocoon, elongated-shaped "Liniya 27 " system were selected.

While the "Marvarid" breed is a component of the industrial hybrids "Golden Valley 1 " and "Golden Valley 2" regionalized in the Republic of Uzbekistan, the "Liniya 27" system is part of the industrial hybrids "Musaffo Tola 1" and "Musaffo Tola 2". The purpose of selecting these breeds for our experiments is that the results obtained in the future will be able to be put directly into practice.

In order to study the lifespan of the female butterflies in detail, during the 2015 worm feeding season, the female butterflies of the "Marvarid" and "Liniya 27" system were 
mixed and isolated from the male butterflies in special round two-sided open ceramic containers (Figure 1).
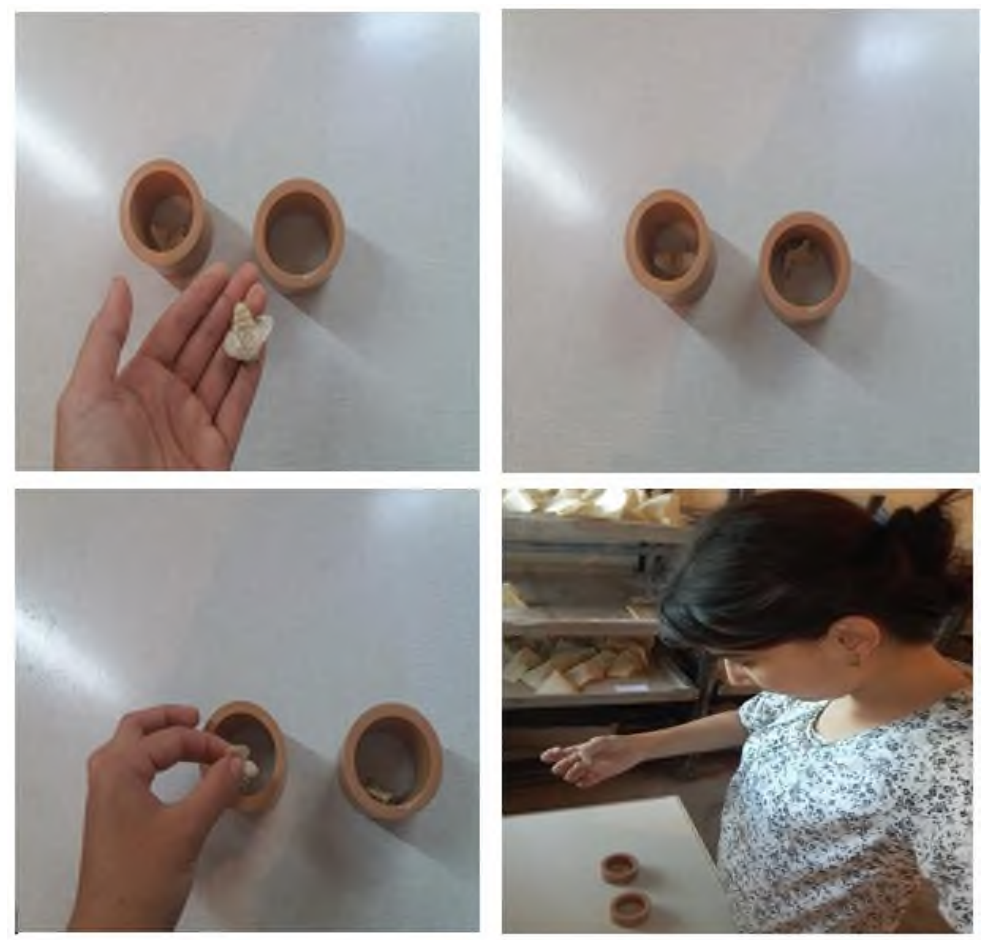

Fig. 1. Insulation on both sides open ceramic pots

The number of butterflies was placed on the parchment paper at the bottom of each container and the survival of the butterflies was monitored individually at the same time each day. The date of death of the butterflies was recorded in the workbook and the days of their stay were determined.

The experimental butterflies were kept in a special room until the end of the estivation period and microanalysis was performed to check for infection. According to the results of microanalysis, each egg yolk found to be healthy was stored in the refrigerator for the winter at a temperature of $+3-4^{\circ} \mathrm{C}$.

\section{Results and discussion}

Table 2 shows the life expectancy of female butterflies and the coefficient of variability of this character. 
Table 2. Lifespan of female 'Marvarid' and 'Liniya 27' species (2015)

\begin{tabular}{|c|c|c|}
\hline & $\begin{array}{c}\text { Life expectancy of female } \\
\text { butterflies }\end{array}$ & \\
\hline $\bar{X}_{ \pm \mathbf{S}} \bar{x}$, day & \\
\hline 'Marvarid' & $10.9 \pm 1.95$ & \\
\hline 'Liniya $27 '$ & $12.8 \pm 0.99$ & 56.6 \\
\hline Average & $\mathbf{1 1 . 9}$ & $\mathbf{4 2 . 3}$ \\
\hline
\end{tabular}

Before analyzing the figures in Table 1, it should be noted. Some literature suggests that female butterflies can live for 2-3 days to 20 days, and that this trait is considered insignificant in terms of selection. However, extensive research has shown that there is a correlation between the body size of female butterflies and the characteristics of the leading economic value. In the selection work based on the motor activity of male butterflies, it was proved that it is possible to obtain a generation with a high viability. In addition, experimental results have been published on the fact that the survival rate of worms in the offspring obtained from genotypes of Strunnikov male butterflies with rapid copulation is increased by $20-30 \%$. Based on this, the feasibility of the life expectancy feature of female butterflies has been the focus of our research work [10, 12, 13].

In our initial results, the lifespan of female butterflies on the "Marvarid" and "Liniya 27 " system was $10.9-12.8$ days. The variability of this trait is in the range of $33.9-56.6 \%$. The coefficient of variability $(\mathrm{Cv})$ in a population indicates the degree of variability or diversity of that trait, and its level indicates whether there is a basis for selection in a selection population. The detected variability coefficient showed that there was a very wide range of variations in the population of "Marvarid" and "Liniya 27' breeds. Thus, the results obtained showed that in the population of these two breeds there is an opportunity to carry out selection work on the sign of life expectancy of female butterflies.

One of the main tasks of the research work is to determine the degree of correlation between the life expectancy of female butterflies and the characteristics of the leading farm value. During the selection and breeding work, the correlation between the characters of economic value is of great importance. This is because the selection process performed on a particular character should not lead to a decrease in another secondary character. In addition, knowledge of the directions of interrelation of important traits is of great scientific and practical importance in the development of the selection program and the correct choice of selection methods.

In this regard, we sought to determine the level of correlation between the life expectancy and productivity, viability, and technological traits of female butterflies in the 27 "Marvarid" and "Liniya 27" species, which differed sharply from each other.

To do this, in 2015, the species were divided into three different groups - gradations on the life expectancy of female butterflies.

-The longest-lived butterflies in the gradation (16-24 days);

-The most average-lived butterflies in gradation (9-11 days); 
The least lived butterflies (5-6 days) were included in the 3rd gradation. According to the results of microanalysis on these gradations, egg yolks found to be healthy were combined and egg yolk mixtures were prepared.

In the spring of 2016, the gradations were incubated and revived separately. The resurrected worms were fed, the cocoons were removed, and their weight and silkiness were determined. After that, the technological parameters of the cocoons were determined, and the average values of all the characters were calculated by gradations.

The average life expectancy of butterflies determined by gradations, as well as the phenotypic correlation coefficients between the signs of viability, productivity was determined. Phenotypic correlation coefficients were calculated in Microsoft Office Excel. The correlation coefficients obtained are shown in Table 3.

Table 3. Relationship between butterfly life expectancy and selection traits

\begin{tabular}{|c|c|c|c|}
\hline \multirow{2}{*}{$\#$} & \multirow{2}{*}{ Interlinked characters } & \multicolumn{2}{c|}{ Correlation coefficient $\left(\mathbf{r}_{\mathbf{p}}\right)$} \\
\cline { 3 - 4 } & & 'Marvarid' & 'Liniya 27' \\
\hline 1 & Lifespan of a butterfly - revival of the eggs & -0.977 & -0.984 \\
\hline 2 & Lifespan of a butterfly - viability of worms & -0.927 & -0.930 \\
\hline 3 & Lifespan of a butterfly - disease percentage & 0.985 & 0.900 \\
\hline 4 & Lifespan of a butterfly - silk cocoon weight & -0.857 & -0.957 \\
\hline 5 & Lifespan of a butterfly - silk cocoon shell weight & -0.861 & -0.706 \\
\hline 6 & Lifespan of a butterfly - silkability & -0.912 & -0.490 \\
\hline
\end{tabular}

Based on the numbers in Table 3, the following points can be made. The "Marvarid" and "Liniya 27" strains showed almost the same result - a very strong negative correlation correlation between the life expectancy of female butterflies and the resuscitation of eggs ( $\mathrm{r}$ $=-0.977 ; \mathrm{r}=-0.984)$.

Between the lifespan of butterflies and the lifespan of worms, very close numbers were obtained for both breeds, and the figure was $r=-0.927 ; r=-0.930$. It is clear from the figures obtained that there is a positive correlation between the incidence rate and the life expectancy of butterflies, in contrast $(r=0.985 ; r=0.900)$. These figures suggest that the shorter the lifespan of female butterflies, the higher the viability of their offspring and the less likely they are to develop disease.

The main goal of our research is to study the effect of life expectancy of female butterflies on cocoon productivity traits. Based on this, we calculated the correlation coefficients between the life expectancy of the female butterflies and the cocoon weight in the two-breed populations. Based on the results obtained, we were convinced that there was a negative or inverse relationship between cocoon weight and butterfly life expectancy. This figure was $r=-0.857$ in the "Marvarid" breed and $r=-0.957$ in the "Liniya 27" breed.

Similar inverse correlation coefficients were found between butterfly life expectancy and cocoon shell weight. In the "Marvarid" breed $\mathrm{r}=-0.861$; "Liniya 27 " zotidar $=$ 0.706. Now, if we analyze the correlation between cocoon silk and butterfly life expectancy, which are the most important among cocoon productivity traits, it was found at $\mathrm{P}=0.490$ in "Liniya 27 ", while $\mathrm{r}=-0.912$ in "Marvarid". This means that the shorter the lifespan of female butterflies, the higher the silkworm characteristics of their offspring. It should be noted that the interdependence of these traits has a negative value in the population of two sharply different breeds, but in "Liniya 27" the line deviates 0 to the value of the "Marvarid" breed. It is this situation that suggests that the interdependence of 
the traits we are studying is directly related to the genotype of the breed, and this problem requires further in-depth study involving breed and selection systems with many different characteristics.

Before analyzing the sign of life expectancy of female butterflies of mulberry silkworm, it is worth recalling some conclusions made by E. Mikhailov and E. F. Poyarkov on the basis of extensive in-depth scientific research [7]. According to senior researchers, healthy butterflies can live from one to two days to 20-25 days after laying eggs. This situation has been confirmed in our experiments. That is, some butterflies of the "Marvarid" and "Liniya 27 ' breeds did not die for 24 days. Based on the results of their experiments and biochemical analysis, these authors concluded that the natural cause of death of butterflies is the spread of intoxication in the body as a result of the breakdown of protein molecules in healthy butterflies. If the body has accumulated enough protein reserves, it means that the effect of many toxins in such an organism will be strong and noticeable.

At this point, V.A. Strunnikov's scientific work on the selection of some rapidly evolving genotypes of male butterflies is noteworthy [8]. That is, according to the author, the ability to rapidly populate sperm in the copulation sac by mixing female and male butterflies can increase the viability of the offspring derived from them by up to $30 \%$. This fact led the author to develop a method for the selection of male butterflies on the rate of copulation in the selection process and their effective use in improving the viability of the breeding generation.

\section{Conclusion}

Based on the goals and objectives of our research work, we determined the life expectancy of female butterflies after spawning to determine the sign of life expectancy. we chose the system. We decided to use such breeds so that the results obtained did not apply only to the same breed. These two breeds and systems were created by scientists of the Breeding Work Laboratory of the Silk Research Institute and are the regionalized Golden Valley 1, Golden Valley 2 and Musaffo Tola 1 and Musaffo Tola 2 industrial hybrids that are undergoing state testing.

In biology, there are some scientific views about the existence of an inverse relationship between the intensity (intensity) of life processes of living organisms and life expectancy. The rapid copulation feature of male butterflies and the short lifespan of butterflies may also be an indication of the presence of a strong energy potential in the body and the intensity of vital processes.

The results of our research in two different breeds prove that the reproduction of female butterflies, which lived relatively less in the selection process of mulberry silkworms, can further increase the signs of viability and cocoon productivity in the next generation.

Butterflies will need to be tested on the basis of modern biochemical analyzes in order to clearly understand the reasons for the higher viability and productivity traits in the offspring of relatively short-lived butterflies.

\section{References}

1. E. L. Westerman, N. W. VanKuren, D. Massardo, A. Tenger-Trolander, W. Zhang, R. I. Hill, M. R. Kronforst, Current Biology 28(21), 3469-3474 (2018)

2. D. Muller, B. Elias, L. Collard, C. Pels, M. J. Holveck, C. M. Nieberding, PloS one 14(11), e0225003 (2019)

3. M. G. Freedman, H. Dingle, S. Y. Strauss, S. R. Ramírez, Proceedings of the National Academy of Sciences 117(46), 28887-28893 (2020) 
4. A. J. Balmer, P. M. Brakefield, O. Brattström, E. van Bergen, Oikos 127(12), 18121821 (2018)

5. E. Burdfield-Steel, M. Brain, B. Rojas, J. Mappes, Oikos 128(2), 245-253 (2019)

6. G. Kumar, M. Khan, J Entomol Zool Stud 6(3), 444-447 (2018)

7. B. U. Nasrillaev, K. S. Giyasova, M. SH. Jumaniyozov, Method for testing the technological properties of cocoons by the graininess of the silk shell and the compactness of cocoons in the silkworm, 46 (Navruz Press, Tashkent, 2015)

8. B. U. Nasrillaev, Fundamentals of genetic selection of mulberry silkworm (Bombyx mor L) aimed at increasing the productivity and technological properties of cocoons, 341 (Samarkand State University, Samarkand, 2018)

9. P. Potocký, A. Bartoňová, J. Beneš, M. Zapletal, M. Konvička, Insect Conservation and Diversity 11(5), 493-505 (2018)

10. N. S. Navruzov, M. Khalilova, U. S. Umarova, Agro-knowledge 1(33), 35-36 (2015)

11. E. Coulthard, J. Norrey, C. Shortall, W. E. Harris, Biological Conservation 233, 213219 (2019)

12. S. Navruzov, U. S. Umarova, Agro-knowledge 6(50), 66-68 (2017)

13. U. S. Umarova, S. Navruzov, F. Jumaeva, Agro-knowledge 3(47), 50-51 (2017)

14. N. Normamatova, O. Hojiev, S. Navruzov, J. Zooveterinary 7-8, 44-45 (2012) 